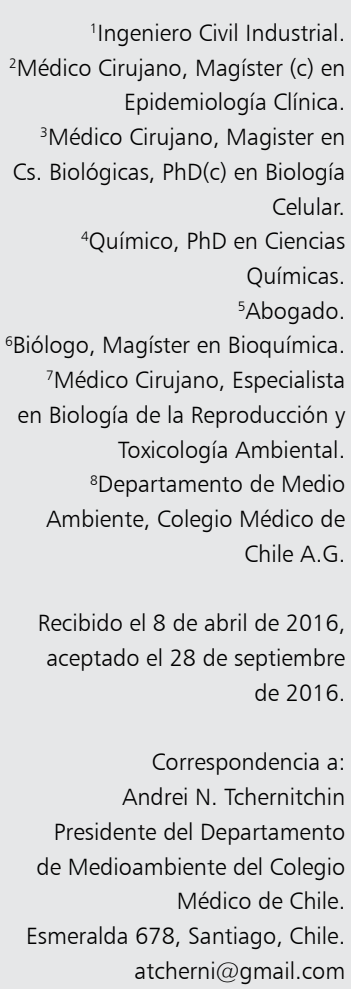

${ }^{1}$ Ingeniero Civil Industrial.

${ }^{2}$ Médico Cirujano, Magíster (c) en Epidemiología Clínica. ${ }^{3}$ Médico Cirujano, Magister en Cs. Biológicas, PhD(c) en Biología Celular.

${ }^{4}$ Químico, PhD en Ciencias Químicas. ${ }^{5}$ Abogado.

${ }^{6}$ Biólogo, Magíster en Bioquímica. ${ }^{7}$ Médico Cirujano, Especialista en Biología de la Reproducción y Toxicología Ambiental. ${ }^{8}$ Departamento de Medio Ambiente, Colegio Médico de Chile A.G.

Recibido el 8 de abril de 2016, aceptado el 28 de septiembre de 2016.

Correspondencia a: Andrei N. Tchernitchin Presidente del Departamento de Medioambiente del Colegio Médico de Chile. Esmeralda 678, Santiago, Chile. atcherni@gmail.com

\section{Consecuencias de la fluoración del agua potable en la salud humana}

\author{
VERENA ROMERO ${ }^{1,8}$, FRANCES J. NORRIS ${ }^{2,8}$, \\ JUVENAL A. RÍOS ${ }^{3,8}$, ISEL CORTÉS ${ }^{4,8}$, ANDREA GONZÁLEZ ${ }^{5,8}$, \\ LEONARDO GAETE ${ }^{6,8}$, ANDREI N. TCHERNITCHIN ${ }^{7,8}$

\section{The impact of tap water fluoridation on human health}

The purpose of this review is to describe the osteological, neurological, endocrine and dermatological effects of fluoride ingestion. Additional aims are to evaluate whether the Chilean tap water fluoridation program has had any impact on dental health, and analyze the basis for the Chilean elementary school milk fluoridation program, which is targeted at children living in places where tap water has a fluoride concentration less than $0.3 \mathrm{mg} / \mathrm{L}$, without any artificial fluoridation process. We discuss the finding that both public measures have no direct or remarkable effect on dental health, since topical dental hygiene products are the main and most effective contributors to the prevention of dental decay. We also suggest that the permanent and systematic ingestion of fluorides imposes health risks on the population. Therefore, we recommend reevaluating the national fluoridation program for public tap water and the elementary school milk program.

(Rev Med Chile 2017; 145: 240-249)

Key words: Dental Health Surveys; Fluoridation; Fluorides.

\section{Consideraciones generales}

\section{Fuentes de exposición e ingestión de fluoruro}

Tr as fuentes de exposición e ingestión de fluoruro son, principalmente: agua potable $\int$ fluorada, pastas, barnices y otros productos dentales, té, pescados, mariscos, sal y alimentos infantiles. El fluoruro ( $\left.\mathrm{F}^{-}\right)$también se encuentra en medicamentos, anestesias, plaguicidas, desechos industriales (aluminio, fertilizantes y minerales de hierro), y como halogenado más abundante en la corteza terrestre y océanos, que lo liberan al ambiente'.

\section{Mecanismos de interacción del fluoruro en el esmalte y la dentina}

El fluoruro es el elemento químico más electronegativo. El ion fluoruro forma compuestos $\mathrm{y}$ reacciona con la mayoría de moléculas orgánicas e inorgánicas.

El esmalte y la dentina están formados por hidroxiapatita (fosfato de calcio) que es disuelta por los ácidos de la descomposición bacteriana, de los alimentos ingeridos. Los iones fluoruro forman fluoroapatita con el esmalte dental, que es menos soluble en los ácidos que la hidroxiapatita, otorgando mayor resistencia al esmalte. El fluoruro de calcio es su principal fuente natural y se caracteriza por su baja solubilidad en agua ${ }^{2}$.

El fluoruro en la prevención de caries comenzó en 1945, con la fluoración del agua potable de la comunidad de Grand Rapids, de EE. UU, con $1 \mathrm{mg} / \mathrm{L}^{1}$. Posteriormente, la acción tópica del fluoruro en productos dentales fundamentó su utilización y masificación.

En adelante, según el Sistema Internacional de Unidades, se usará $1 \mathrm{mg} / \mathrm{L}$ como equivalente a 1 ppm. 


\section{Programas de fluoración masiva en Chile}

\section{Fluoración del agua potable}

Chile es pionero en la fluoruración del agua potable, desde que en 1953 implementara un programa piloto de fluoruración en Curicó. En 1958 fue ampliado a 73 comunidades, cubriendo el 58\% del país, con fondos internacionales. En 1977 se suspendió, por falta de recursos ${ }^{3}$. Posteriormente, en 1986, se restableció, en Valparaíso, implementándose, progresivamente, en 14 de 15 regiones, excluyendo la Región del Bío-Bío ${ }^{4}$.

En Chile, la concentración óptima de flúor recomendada en agua potable, sea natural o artificial, oscila entre 0,6 y $1,0 \mathrm{mg} / \mathrm{L}$, con límite máximo de $1,5 \mathrm{mg} / \mathrm{L}^{3}$. Se estima que la cobertura nacional de agua potable fluorada alcanza un $82,3 \%{ }^{5}$.

Algunos autores señalan que el consumo de agua fluorada en concentraciones óptimas reduce la prevalencia de caries dentales, pero durante el período de desarrollo dentario puede aumentar la prevalencia de fluorosis dental en grados leves ${ }^{7,8}$, observado en áreas fluoradas como no fluoradas. La ingesta de fluoruro, por otras fuentes, ha aumentado desde 1940, cuando el agua era la fuente principal. El $71 \%$ de estudios de caries que evaluaron la fluoración del agua son previos a 1975, cuando se masificó el uso de productos dentales, excluyéndose estudios de caries en adultos".

Dado que la ocurrencia de fluorosis es dosis-dependiente ${ }^{10,11}$ y que la disponibilidad de flúor (F) es muy variable, existe preocupación por posibles efectos adversos en la salud humana.

Otros autores sugieren que la fluoración del agua no reduce la prevalencia de caries dentales. Estudios recientes indican ausencia de diferencias del número de caries dentarias entre niños que toman agua fluorada versus sin fluorar ${ }^{12}$. Peckham y Awofeso concluyeron que la evidencia sugiere que el fluoruro tiene la potencialidad de generar problemas en salud, mientras que sólo presenta un discreto efecto en prevención de caries dentales ${ }^{13}$.

\section{Fluoración de la leche, en escuelas sin agua} potable fluorada

El Programa de Alimentación Escolar (PAE), que entrega leche fluorada a escolares de $1^{\circ}$ a $8^{\circ}$ básico, se aplica a escuelas rurales que no poseen agua fluorada $(<0,3 \mathrm{mgF} / \mathrm{L})$.

La concentración recomendada de fluoruro en la leche es de 3,15 a 4,25 mg/L. Por lo tanto, la cantidad de F-aportado por porción $(200 \mathrm{~mL})$ de consumo habitual de leche es de 0,63-0,85 $\mathrm{mg}^{3}$, entregada al desayuno, almuerzo, once, colación y cena, según corresponda.

\section{Fluorosis dental, en adolescentes}

En 2007, un levantamiento epidemiológico realizado en Chile, reveló que el 31,2\% de adolescentes de 12 años presentan algún tipo de fluorosis, principalmente en grados leves y muy leves ${ }^{14}$.

Cerca de 3 millones de niños chilenos consumen leche fluorada, diluida con agua que naturalmente posee al menos $0,3 \mathrm{mg} / \mathrm{L}$ de F-. Si se considera que al menos consumen 3 vasos de leche al día de $200 \mathrm{ml}$, la ingesta de F-sería de 2,59$3,6 \mathrm{mg} /$ día, por sobre cualquier recomendación internacional, como única fuente deF- .

\section{Aspectos legales de la política de fluoración del agua potable}

La fluoración del agua potable en Chile obliga a los ciudadanos al consumo, involuntario, de un químico que no, necesariamente, requieren. Por décadas, la mayoría de la población chilena ha sido sobreexpuesta a este elemento, potencialmente no inocuo para la salud, transgrediendo las garantías constitucionales.

\section{Metodología}

Se efectuó una revisión de estudios científicos en Medline (Pubmed) e ISI, con citas relativas a posibles efectos por exposición a flúor. Se seleccionaron los estudios que por metodología (control de variables confundentes) y relevancia, fueran por sí mismos, irrefutables. Ante controversia, se citan estudios de ambas partes, avalados por su solidez estadística. Estudios que no encuentran una relación causa-efecto, no se describen mayormente, si han sido criticados por otras publicaciones por falta de control de variables.

\section{Efectos en la salud por ingesta y exposición crónica al fluoruro}

\section{Efectos osteo-esqueléticos}

Un estudio epidemiológico demostró que la fluoración de agua potable, con 1,0 mg/L, en una comunidad en Utah, Estados Unidos, en comparación a dos comunidades de característi- 
cas semejantes que consumían agua con niveles de fluoruro bajo $0,3 \mathrm{mg} / \mathrm{L}$, reveló un aumento importante de la incidencia fracturas óseas por osteoporosis (especialmente, cadera) en la tercera edad, tanto en hombres como en mujeres ${ }^{15}$. Un estudio, en Finlandia, reveló resultados similares $^{16}$. Se ha relacionado la exposición crónica a niveles más elevados de flúor en agua potable (4 $\mathrm{mg} / \mathrm{L}$, en comparación con $1 \mathrm{mg} / \mathrm{L}$ ) con el doble de fracturas de cadera en personas de edad ${ }^{17,18}$. En concordancia con lo anterior, los estudios de Riggs y cols. confirman que, aún con la terapia para osteoporosis en mujeres postmenopáusicas, altas dosis de fluoruro aumentan el porcentaje de hueso esponjoso y disminuye el hueso compacto cortical, aumentando la fragilidad y fracturas óseas, con la excepción de las vertebrales ${ }^{19}$. No obstante lo anterior, otros estudios han atribuido el incremento de facturas a un descenso del fluoruro a niveles inferiores a los habituales ${ }^{20}$.

Numerosos autores no han demostrado diferencias significativas como efecto de niveles diferentes de fluoruro en agua potable ${ }^{21}$, incluyendo un meta-análisis, $y$ atribuyen las diferencias entre los trabajos a la alta presencia de factores confundentes ${ }^{22}$.

\section{Efectos neurológicos}

Desde 1975, estudios en animales investigan la toxicidad del fluoruro en distintos órganos, particularmente en cerebro. La respuesta tisular de enzimas del metabolismo intermediario, en estudios iniciales, no mostró diferencias significativas $^{23}$. Estudios posteriores reportaron que de 36 pacientes con fluorosis, 16 de ellos mostraron una discreta alza del anión en el líquido cefaloraquídeo, sugiriendo la alta selectividad y capacidad de autorregulación de la barrera hematoencefálica frente a estos iones ${ }^{24}$. Estudios en animales demostraron que una exposición crónica a fluoruros, lo acumulan en el hipocampo e hipotálamo ${ }^{25,26}$. En un estudio con ratas Wistar tratadas con dosis altas de fluoruros, por 60 días con $20 \mathrm{mg} / \mathrm{L}$ de fluoruro sódico $(\mathrm{NaF})$, reportó una disminución del peso cerebral, deterioro axonal, vacuolización de las fibras de mielina y edema mitocondrial, demostrando signos neurodegenerativos en cerebro, médula espinal y nervio ciático ${ }^{27}$.

En estudios epidemiológicos realizados en Carolina del Norte, con dosis muy reducidas de $\mathrm{F}^{-}$, demostraron que la incidencia de demencia primaria tipo Alzheimer, era mayor en zonas donde el contenido de fluoruro en el agua era mayor ${ }^{28}$. Otro estudio demostró que en Etiopía (1976-1985), donde la población consumió agua fluorada sobre $4 \mathrm{mg} / \mathrm{L}$, la incidencia de mielopatía sin radiculopatía fue de un $28 \%$, y con radiculopatía fue de un $72 \%{ }^{29}$.

Un meta-análisis de 27 estudios epidemiológicos, concluyó que la exposición crónica al fluoruro del agua afectaba al neurodesarrollo de los niños y su coeficiente intelectual (CI), recomendando estudiar la exposición prenatal a este elemento ${ }^{30}$. Otro estudio demostró que el porcentaje de niños con fluorosis dental estaba más en la categoría de CI extremadamente bajo y bajo; en cambio el porcentaje de niños sin fluorosis dental, estaba más en la categoría de CI medio o alto ${ }^{31}$. En estudio de una localidad de China, con $\mathrm{F}$ en agua potable promedio de $1,3 \mathrm{mg} / \mathrm{L}$, demostró que cada $1 \mathrm{mg}$ F/L en orina el CI disminuye 0,59 puntos; y que existe una relación dosis-respuesta entre el $\mathrm{F}$ en orina y la fluorosis dental, al igual que con el $\mathrm{CI}^{32}$. Otros estudios no han encontrado correlaciones entre el F y deterioros en salud ${ }^{33,34}$.

\section{Efectos endocrinos}

En el Reino Unido, cerca del 10\% de la población recibe agua florada con $1 \mathrm{mg} / \mathrm{L}$, apoyado por un informe de salud pública que concluyó que la medida era adecuada ${ }^{35}$, aunque, estudios previos habían sugerido la correlación entre consumo de fluoruro e hipotiroidismo ${ }^{36}$.

Los efectos del fluoruro sobre la tiroides han sido descritos por largo tiempo ${ }^{37}$. En la década del 50, el fluoruro se usaba para reducir la actividad de la tiroides en pacientes con hipertiroidismo. Se propuso que el fluoruro un inhibidor de la función tiroidea, basados en estudios de su relación con el bocio. Además, las terapias con fluoruro redujeron la actividad tiroidea en varios pacientes ${ }^{38}$. Se encontró que dosis entre 2 y $5 \mathrm{~kg} / \mathrm{L}$ por día, eran efectivos para tratar hipertiroidismo, lo que corresponde a las dosis en agua potable de áreas fluoradas ${ }^{39-41}$.

Dos trabajos concluyeron que el fluoruro es un disruptor endocrino con el potencial de alterar la función de tejidos que requieran yodo ${ }^{37}$. Se sugirió que su efecto era disminuir la función tiroidea, con exposición a $\mathrm{F}$ de $0,01 \mathrm{mg} / \mathrm{kg} /$ día cuando la ingesta de yodo fuera inadecuada ${ }^{42}$.

En un estudio en Inglaterra, se encontró una 
mayor prevalencia de hipotiroidismo $(30 \%)$ en áreas con fluoruro entre $0,3 \mathrm{mg} / \mathrm{L}$ y $0,7 \mathrm{mg} / \mathrm{L}$, comparados con áreas bajo $0,3 \mathrm{mg} / \mathrm{L}$, y una prevalencia, aún, mayor donde los niveles de fluoruro eran $>0,7 \mathrm{mg} / \mathrm{L}$, sin considerar otras fuentes de flúor ${ }^{43}$.

\section{Efectos dermatológicos}

Experimentalmente, Prado y cols. demostraron en una línea de queratinocitos ( $\mathrm{HaCaT})$, que altas dosis de fluoruro retardaban la diferenciación de estas células, sin alterar el patrón de expresión de queratina ${ }^{44}$.

Clínicamente, se ha descrito el concepto de fluoroderma como una reacción de hipersensibilidad asociada a exposición a compuestos fluorados. Se caracteriza por la aparición de lesiones pápulo-nodulares en la región perioral y en la frente, que pueden extenderse hacia cuello, tórax y dorso. Ocasionalmente, puede presentarse con placas exudativas, nódulos y úlceras malignas fungiformes necróticas.

La exposición tópica a elementos fluorados aumenta el riesgo de desarrollo de fluoroderma ${ }^{45}$.

En la Figura 1 se ilustran los impactos que el fluoruro genera en la salud humana.

\section{Comparación de políticas públicas internacionales en la fluoración del agua potable}

\section{Fluoración del agua potable en el mundo}

A nivel mundial la fluoración del agua potable, de manera artificial, afecta sólo a un 5,7\% de la población, según el estudio de Cheng et al. ${ }^{46}$.

La recomendación de la OMS y la Agencia de Protección del Medio Ambiente para fluoración del agua potable, por décadas, fue de $1,5 \mathrm{mg} / \mathrm{L}$, sin embargo, en el 2008, este límite, definido como seguro para evitar la fluorosis dental, pero "óptimo" para prevenir deterioros dentales, disminuyó a 0,5-1,0 mg/L (Tabla 1A). Sin embargo, muchos países continúan sin ajustarse (Tablas 1B, 1C, 1D).

Contrariamente, el 98\% de los países europeos prohiben fluorar el agua potable (Tabla 2A, 2B, 2C). La mayoría ha definido la política de fluorar algunas sales o disponer de píldoras o tabletas fluoradas, sólo para los niños que lo requieran.

\section{Evolución de los deterioros dentales}

Según datos de la OMS, entre 1970 y 2013, observamos una disminución progresiva, y a la misma tasa, del deterioro de piezas dentales en ni-

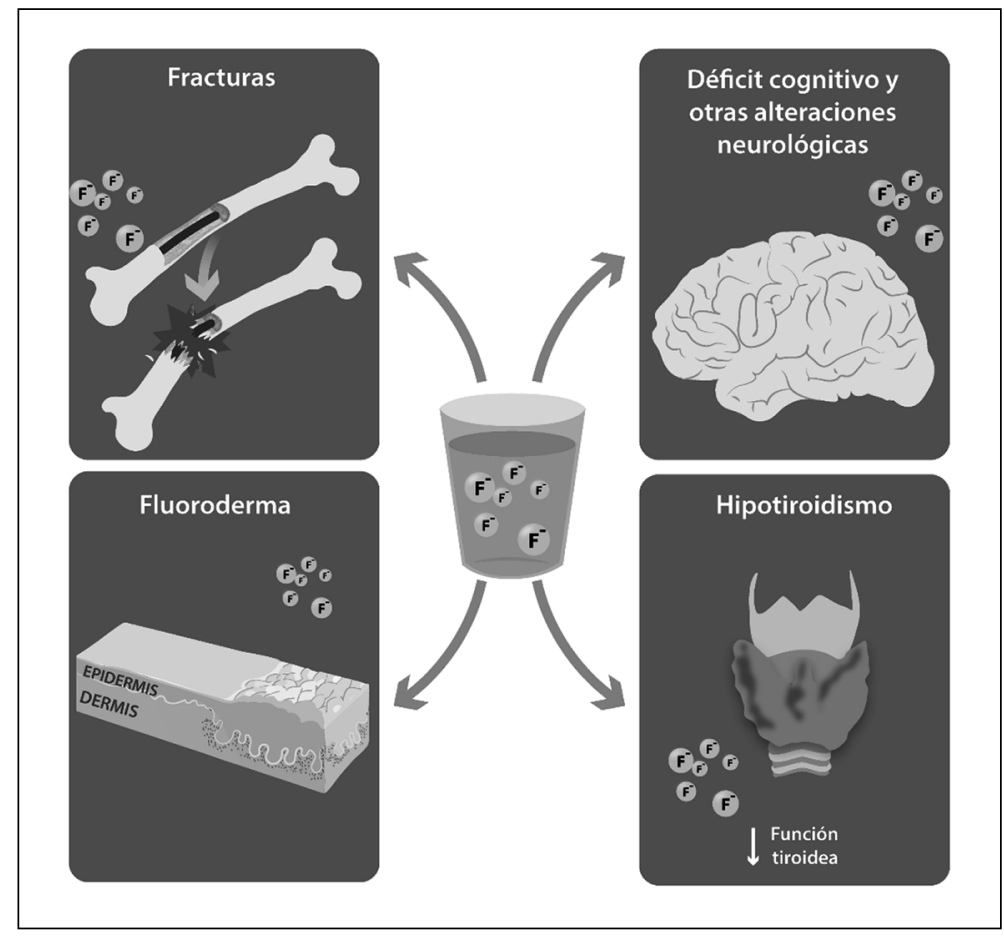

Figura 1. Posibles efectos del Fluoruro $\left(F^{-}\right)$en el organismo. Cuadrante superior izquierdo; muestra que la ingesta de $F^{-}$pudiera aumentar el riesgo de fracturas en la edad adulta por trastornos en la calcificación. Cuadrante inferior izquierdo; muestra lesiones a nivel de la dermis, conocidas como fluoroderma. Cuadrante superior derecho; muestra posibles efectos adversos del $\mathrm{F}^{-}$a nivel del sistema nervioso central resultando en déficit cognitivo. Cuadrante inferior derecho; la ingesta de $\mathrm{F}$, puede podría reducir la función tiroidea. 
Tabla 1. Límites máximos de fluoruro en agua potable, en $\mathrm{mg} / \mathrm{L}$, de la OMS y algunos países de América

A.

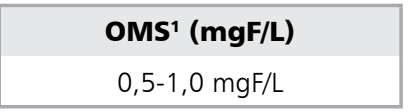

B.

\begin{tabular}{|c|c|c|}
\hline \multicolumn{3}{|c|}{ Norteamérica (mgF/L) } \\
\hline & & Canadá \\
\hline EPA $^{2}$ & $\mathrm{CDC}^{3}$ & \\
\hline $2,0-4,0$ & $0,7-1,2$ & $0,7-1,5$ \\
\hline
\end{tabular}

C.

\begin{tabular}{|c|c|c|c|}
\hline \multicolumn{4}{|c|}{ Centroamérica (mgF/L) } \\
\hline Guatemala $^{5}$ & México $^{6}$ & El Salvador? & Costa Rica ${ }^{8}$ \\
\hline 1,7 & 0,7 & 1,0 & $0,7-1,5$ \\
\hline
\end{tabular}

D.

\begin{tabular}{|ccccccc|}
\hline & \multicolumn{4}{c}{ Sudamérica (mgF/L) } & & \\
Argentina $^{9}$ & Ecuador & Colombia & Bolivia & Chile $^{\mathbf{1 0}}$ & Brasil $^{\mathbf{1 1}}$ & Uruguay \\
$0,8-1,7$ & 1,7 & 1,5 & 1,5 & 1,5 & 1,5 & 0,0 \\
\hline
\end{tabular}

${ }^{1}$ La OMS, en el 2008, recomendó 0,5-1,0 mg/L para la fluoración del agua potable. Anteriormente, el límite era 1,5 mg/L. ${ }^{2}$ Para evitar fluorosis dental, el límite legal ejecutable es 2,0. \% según CDC al año 2010. ${ }^{3} \mathrm{CDC}$ recomienda nuevo límite de 0,7 mg/L el 27 de abril de 2015, para prevenir fluorosis dental. ${ }^{4}$ En el 2008, el Panel de Expertos en Fluoruro recomendó usar 0,7 mg/L. Antes era entre 0,7 y 1,0, sin embargo, el límite máximo se dejó igual en 1,5 mg/L. ${ }^{5}$ Límite máximo permisible COGUANOR 29001:99 ${ }^{6} \mathrm{En}$ el caso de México, el límite máximo de fluoruro en agua es de 0,7 mg/L; cuando las concentraciones sobrepasan este valor, no se debe consumir sal yodada-fluorada, de acuerdo con la norma NOM-013-SSA2-2006 (SSA 2007). ${ }^{7}$ Norma Salvadoreña Obligatoria NSO 13.07.01:08 AGUA, AGUA POTABLE. (Segunda actualización) Publicada en el Diario Oficial el 12 de junio de $2009 .{ }^{8} 1,5 \mathrm{mg} / \mathrm{L}$ para temperaturas de 8 a $12{ }^{\circ} \mathrm{C}$ y $0,7 \mathrm{mg} / \mathrm{L}$ para temperaturas de 25 a $30{ }^{\circ} \mathrm{C}$. ${ }^{9}$ Límites variables en función de la temperatura media y máxima de la zona. ${ }^{10}$ Decreto Supremo N7a35 del 7 de noviembre de 1969 y su actualización, Decreto Supremo № 131 de 200, p 7. Región del Biobío rechazó la fluoración del agua potable, para prevenir los efectos adversos en salud. ${ }^{11}$ Ley Federal y de Estados definió máx F como $1 \mathrm{mgF} / \mathrm{L}$, pero el Ministerio de Salud acepta como máximo $1,5 \mathrm{mg} / \mathrm{L}$.

ños de 12 años, tanto en países que fluoran el agua potable como en los que no la fluoran (Figura 2).

Análogamente, el índice DMFT en los países que no fluoran el agua potable y no usan sal fluorada, comparado con los que utilizan fluoración, a través de la sal o tabletas, la tendencia es a la disminución, acercándose a 1 pieza deteriorada, en ambos casos (Figura 3).

Por lo tanto, la fluoración del agua potable y las sales no tienen incidencia alguna, en la disminución de deterioros dentales. Los mejores hábitos de higiene y alimentación (reduciendo carbohidratos) siguen siendo las más efectivas.

\section{Programa de leche fluorada como sustituto del agua potable fluorada y fluoruros tópicos}

Según, la OMS una alternativa eficaz para prevenir deterioros dentales en niños, es la fluoración de la leche y otros productos lácteos. Esto puede ser fundamentado en Chile por dos estudios: a. Codegua, Chile (1994-1997), con dosis de 0,25 $\mathrm{mg}$ F/día en la leche para niños entre 1-2 años y 0,75 mg F/día para 3-6 años, sin interrumpir el uso pasta dental dos veces al día, se observó una disminución de deterioros dentales de un 40\% (niños de 4 años) y de un 78\% (niños de 5 años).

b. Araucanía, Chile (1999-2002), con niños de 9-12 años, tomando leche fluorada con 0,65 mg F/día, con una concentración promedio de 3,13 mg F/L se observó una reducción del 24-27\% de deterioros dentales, sin interrumpir el uso de pastas dentales, diariamente. Al final del estudio, un 39\% de los niños de 9 años no tenían deterioros dentales (DMFT $=0)$ comparados el 21\%-23\% inicial. Los niños de 12 años no mostraron deterioros dentales, comparados con el $11 \%$ inicial. Al compararlos con los resultados del programa de aplicación tópica de APF (Acidulated Phosphate Fluoride) gel, no fueron muy diferentes; concluyéndose que la fluoración de la leche era un buen sustituto del programa de aplicación de fluoruros tópicos ${ }^{47}$. 
Tabla 2. Límites máximos de fluoruro en agua potable, en mg F/L, y su sustituto para la prevención de deterioros dentales en algunos países de Europa

\begin{tabular}{|c|c|c|c|c|c|c|c|c|}
\hline \multirow[t]{5}{*}{ A. } & País & Austria $^{1}$ & Bélgica $^{2}$ & \multicolumn{2}{|c|}{ Dinamarca $^{3}$} & Finlandia ${ }^{4}$ & Francia $^{5}$ & Alemania $^{6}$ \\
\hline & $\mathrm{F}$ en agua potable $\mathrm{ppm}=\mathrm{mgF} / \mathrm{L}$ & 0,0 & 0,0 & & 0 & 0,0 & 0,0 & 0,0 \\
\hline & \% del País & 100 & 100 & & 00 & 100 & 100 & 100 \\
\hline & Substituto & $\begin{array}{l}\text { Sal con F- y } \\
\text { Mejor higiene }\end{array}$ & $\begin{array}{l}\text { Mejor } \\
\text { higiene }\end{array}$ & & $\begin{array}{l}\text { ejor } \\
\text { iene }\end{array}$ & $\begin{array}{c}\text { Tabletas y } \\
\text { Mejor higiene }\end{array}$ & $\begin{array}{l}\text { Sal con Fy } \\
\text { Mejor higiene }\end{array}$ & $\begin{array}{c}\text { Sal con Fy } \\
\text { e Mejor higiene }\end{array}$ \\
\hline & $\%$ Sales/Tabl c/F- en el marcado & $6 \%$ & & & & $70 \%$ & $65 \%$ & $67 \%$ \\
\hline \multirow[t]{5}{*}{ B. } & País & Luxemburgo? & \multicolumn{2}{|c|}{ Holanda ${ }^{8}$} & \multicolumn{2}{|c|}{ Noruega9 } & Suecia $^{10}$ & Suiza ${ }^{11}$ \\
\hline & $\mathrm{F}$ en agua potable $\mathrm{ppm}=\mathrm{mg} / \mathrm{L}$ & 0,0 & \multicolumn{2}{|c|}{0,0} & \multicolumn{2}{|r|}{0,0} & 0,0 & 0,0 \\
\hline & \% del País & 100 & \multicolumn{2}{|c|}{100} & \multicolumn{2}{|r|}{100} & 100 & 100 \\
\hline & Substituto & $\begin{array}{l}\text { Sal con F- y } \\
\text { Mejor higiene }\end{array}$ & $\begin{array}{l}\text { Sal co } \\
\text { Mejor } h\end{array}$ & $\begin{array}{l}n F^{-} y \\
\text { igiene }\end{array}$ & Mejo & r higiene & ejor higiene & $\begin{array}{l}\text { Sal con F-y } \\
\text { Mejor higiene }\end{array}$ \\
\hline & $\%$ Sales/Tabl c/F- en el marcado & $75 \%$ & 25 & & & & & \\
\hline \multirow[t]{5}{*}{ C. } & País & \multicolumn{2}{|c|}{ Irlanda ${ }^{12}$} & \multicolumn{3}{|c|}{ Inglaterra $^{13}$} & \multicolumn{2}{|c|}{ España $^{14}$} \\
\hline & $\mathrm{F}$ en agua potable $\mathrm{ppm}=\mathrm{mg} / \mathrm{L}$ & \multicolumn{2}{|c|}{$0,6-0,8$} & \multicolumn{3}{|r|}{1,5} & \multicolumn{2}{|r|}{1,5} \\
\hline & \% del País & \multicolumn{2}{|c|}{$100 \%$} & \multicolumn{3}{|c|}{$10 \%$} & \multicolumn{2}{|c|}{$11 \%$} \\
\hline & Substituto & & & & & & \multicolumn{2}{|c|}{ Sal con F- } \\
\hline & $\%$ Sales/Tabl c/F- en el marcado & & & & & & & $10 \%$ \\
\hline
\end{tabular}

${ }^{1}$ Fluoruros se consideran tóxicos, por lo que nunca se han agregado al agua potable. M. Eisenhut, Head of Water Department, Osterreichische Yereinigung fur das Gas-und Wasserfach Schubertring 14, A-1015 Wien, Austria, February 17, $2000 .{ }^{2}$ Nunca se ha fluorado el agua potable. Fluoruros se venden sólo con prescripción médica, si se requieren, tomando en cuenta las múltiples fuentes de exposición (alimentos, cremas dentales, medicamentos y otros). Chr. Legros, Directeur, Belgaqua, Brussels, Belgium, February 28, 2000. Desde el 8 de julio de 2002 el Decreto Real, elaborado por el Department of Social Affairs, Public Health, and Environment, prohibe la venta en todo el país de suplementos que contengan fluoruros, incluidos chicles y similares. Cualquier suplemento fluorado requiere receta médica. Los productos de higiene bucal se consideran de uso cosmético, por lo que se exceptúan de este requisito. ${ }^{3}$ Fluoruros se consideran tóxicos, por lo que nunca se han agregado al agua potable. Klaus Werner, Royal Danish Embassy, Washington DC, December 22, 1999. “Sólo en Kuopio se fluoró el agua entre 1959 y 1992. Las píldoras para niños son recomendadas por la Asociación de Dentistas de Finlandia desde 1995, cuando se requieran. Cada persona tiene el derecho de tomar agua potable sin químicos adicionados como medicamento para reducidos grupos de población, y bajo alimentación forzada. Leena Hiisvirta, M.Sc., Chief Engineer, Ministry of Social Affairs and Health, Finland, January $12,1996$. ${ }^{5}$ Desde 1985 se autorizó el uso de sal fluorada hasta $250 \mathrm{mg} / \mathrm{L}$, coexistiendo con sal sin F. Pero, no se recomienda su uso si el agua potable natural de la zona, contiene más de 0,5 mg/L de F. Debido a consideraciones éticas y médicas, el fluoruro no está en la lista de aditivos para el agua potable. Louis Sánchez, Directeur de la Protection de l'Environment, August 25, 2000. ${ }^{6} \mathrm{Nunca}$ se ha fluorado el agua potable. Principalmente, porque se considera una forma compulsiva de medicación masiva y porque el 99\% de los desechos tóxicos de los fluoruros usados se devuelven al agua. Gerda Hankel-Khan, Embassy of Federal Republic of Germany, September 16, 1999. ${ }^{7}$ Nunca se ha fluorado el agua potable. Pues, no se considera apropiado un tratamiento medicinal por esa vía. Cada persona puede decidir por sí misma, si necesita tomar pastillas de fluoruro. Jean-Marie RIES, Head, Water Department, Administration De L'Environment, May 3, 2000. ${ }^{\circ}$ Se fluoraba el agua potable entre 1960 y 1970, para prevenir caries. Luego, se prohibió, pues la Corte Suprema concluyó que no existían bases legales. Se trató de corregir las leyes, pero no hubo apoyo Parlamentario. Razón principal: fluoración es un aditivo medicinal al agua, obligado por el Estado "en beneficio de la sociedad". Desde 1973 no hay fluoración del agua. Wilfred Reinhold, Legal Advisor, Directorate Drinking Water, Netherlands, January 15, 2000. ${ }^{9}$ Nunca se ha fluorado el agua potable. En 1980 comenzaron los intensos debates, concluyéndose que cada persona es libre de dar pastillas de fluoruro a sus hijos, usar enjuagues bucales y pastas dentales, si así lo desea para prevenir caries. Truls Krogh \& Toril Hofshagen, Folkehelsa Statens institutt for folkeheise (National Institute of Public Health) Oslo, Norway, March 1, 2000. ${ }^{10}$ Desde 1971 se prohibe fluorar el agua potable, luego de la derogación de la ley en de agua potable de 1962. Gunnar Guzikowski, Chief Government Inspector, Livsmedels Verket-National Food Administration Drinking Water Division, Sweden, February 28,2000 . ${ }^{11}$ Desde 1962, sólo usan sal fluorada. Se realizó un programa con agua fluorada para la ciudad de Basel que tenía ambos sistemas, pero por error los usaban simultáneamente, por lo que se suspendió en el 2003. La suplementación de fluoruro es considerada anti-económica, anti-ecológica, anti-ética (medicación forzada), toxicológica y fisiológicamente contraproducente, pues no se aplica a grupos objetivos, sino se pone en riesgo al resto de la población. Sin considerar su real ingesta, necesidades y limitaciones, pudiendo la excesiva ingesta ser una amenaza de salud para ciertos grupos. Dr. B. Havlik, Ministerstvo Zdravotnictvi Ceske Republiky, October 14, 1999. ${ }^{12}$ Profesores de la Universidad de Kent han alertado que sobre 0,7 mg/L, e incluso sobre 0,3 $\mathrm{mg} / \mathrm{L}$, los casos de hipotiroidismo han aumentado en un 30\% más que en zonas sin F. Por lo que recomiendan detener la fluoración del agua. ${ }^{13}$ En el año 2007, comienza el programa de fluoración de leche para 16.000 niños, del noroeste del Reino Unido. En octubre, 2014 el Ministerio de Salud abandonó el plan de fluorar el agua potable de Southampton y el suroeste de Hamsphire por la oposición de la ciudadanía. ${ }^{14}$ Sobre $1,5 \mathrm{mg} / \mathrm{l}$ las aguas minerales naturales envasadas, deben etiquetarse como no apta para niños lactantes. Además, sobre $1 \mathrm{mg} / \mathrm{L}$ se debe indicar la composición analítica real de $\mathrm{F}$. 

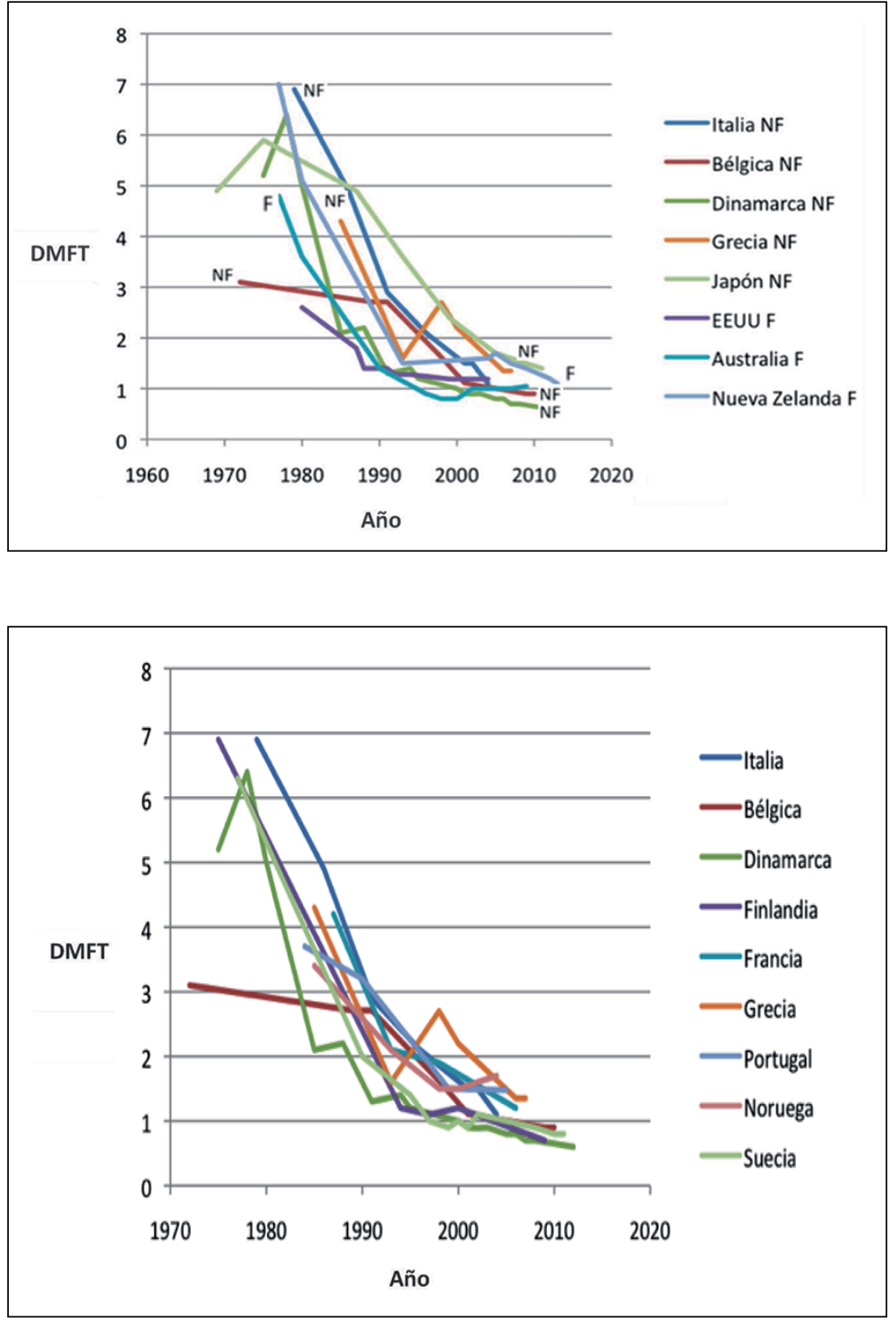

Figura 2. Deterioro dental en países que fluorizan el agua potable vs países que no fluoran el agua potable ni usan sales fluoradas (NF), entre 1970 y 2013. DMFT (Decay, Missing, Filled Tooth) en niños de 12 años. (Datos obtenidos de la OMS, a través del Centro de Colaboración para la Educación, Entrenamiento e Investigación Oral. Universidad de Malmö, Suecia).
Sin embargo, el uso diario de dentífricos, aportaron cientos de veces más flúor que en la leche fluorada. Entonces, los mejores resultados del programa podrían atribuirse sólo al uso sistemático de dentífricos. En particular, si antes no se usaban con la periodicidad definida en los experimentos.

Ambas experiencias son el fundamento del programa de fluoración de la leche escolar, en Chile.

Un estudio realizado en Chile, el año 2010, con 50 niños y niñas, de 8 años, de dos escuelas de Maipú que nunca han recibido leche fluorada, y 40 niños y niñas, de 8 años, de una escuela de Pirque, que recibieron leche fluorada del programa PAE, desde los 6 años, demostró que el índice DMFT (Decay, Missing, Filled Tooth) fue, significativamente, mayor en Pirque $(2,65)$ que en Maipú (1,48). Igualmente, el DMFT (dientes temporales) fue mayor en Pirque $(4,55)$ que en Maipú $(3,82)$. Además, el porcentaje de niños sin caries fue de un $10 \%$ en Maipú y en Pirque fue $0 \%$. Los niños de ambas comunas han residido allí desde su nacimiento, y el agua potable no ha sido fluorada, artificialmente ${ }^{48}$. 
Muchos países europeos que han disminuido, sustancialmente, los deterioros dentales, nunca han tenido programas de fluoración masiva de la leche y derivados (y/o de agua potable).

Por lo tanto, la fluoración de la leche no tiene relevancia alguna en la disminución de los deterioros dentales. Además, los niños que han recibido leches fluoradas, poseen mayor riesgo de desarrollar no sólo fluorosis dental, sino enfermedades como las descritas en esta revisión.

\section{Conclusiones y recomendaciones}

\section{Conclusiones}

a) Los efectos de la ingesta de fluoruros imponen riesgos de diversas enfermedades en el sistema asteo-esquelético, neurológico, endocrino y en la piel. La fluorosis dental y del esqueleto, son signos de ingesta crónica y excesiva de fluoruros.

b) Los infantes, niños y adolescentes, están en alto riesgo de padecer enfermedades por sobre-ingesta de fluoruros, a través del agua potable y/o la leche fluoradas, pues el deterioro de salud es proporcional a la dosis y al tiempo de exposición.

c) La fluoración del agua potable no impacta, significativamente, en la prevención de caries. Pues su eficacia es más bien, por efecto tópico y no sistémico, como demostraron países que no fluoran al agua potable, y no usan leches o sales fluoradas, disminuyendo los deterioros dentales a la misma tasa que los que fluoran el agua potable.

\section{Recomendaciones}

1. Modificar el Decreto $\mathrm{N}^{\circ} 735$ del 7 de noviembre de 1969, su actualización con el Decreto Supremo No 131 de 2006, y el Reglamento de los Servicios Destinados al Consumo Humano de 2007, para evitar la fluoración del agua potable y evitar la fluoración de la leche, en todas las regiones del país.

2. Privilegiar el uso de productos de higiene dental que contengan las cantidades, necesarias, pero mínimas, de fluoruro para mantener la salud dental, reforzar la educación para un mejor cuidado dental y mejor nutrición.

3. Capacitar a los profesionales de educación y de salud, respecto de las consecuencias adversas de la ingesta de fluoruros.
4. Realizar estudios epidemiológicos en Chile, para evaluar los efectos adversos en salud, por ingesta y uso de fluoruros, por décadas.

Agradecimientos y financiamiento: Se agradece al Colegio Médico de Chile A.G. el financiamiento de esta publicación. Se informa que el esquema final, diseñado conceptualmente por los autores, fue encargado y optimizado en su calidad por http://www.illustrative-science.com/.

\section{Referencias}

1. Peckham S, Awofeso N. Water fluoridation: a critical review of the physiological effects of ingested fluoride as a public health intervention. The Scientific World JOURNAL 2014: 293019.

2. Atkins P, Overton T, Rourke J, Weller M, Armstrong F. Química Inorgánica. 4ta Edición, Editores S.A de C.V. McGraw-Hill/Interamericana ISBN; 2008. 970-10-6531$\mathrm{X}$.

3. Ministerio de Salud. Normas de Uso de Fluoruros en la Prevención Odontológica. Norma General Técnica $\mathrm{N}^{\circ} 105$ En: Departamento de Salud Bucal. División de prevención y control de Enfermedades SdSP.; 2008. p. 9, 15,16, 23.

4. Badenier O, Cueto A, Mariño R, Acevedo R. Situación de Salud Bucodental y algunos Factores de riesgo en Adultos de 35 a 44 años de la Región de Valparaíso. Rev Fac Odontología Univ Valparaíso 2009; 1103-8.

5. Gómez S, Fernández O. Fluoración del Agua Potable, Experiencia en Chile. En: Gómez Soler G, Editor. Fluorterapia en Odontología Fundamentos y Aplicaciones Clínicas; 2010. p. 162-77.

6. Decreto Supremo N 735 del 7 de noviembre de 1969 y su actualización, Decreto Supremo No 131 de 2006, p 7. MINSAL. Normas de Uso de Fluoruros en la Prevención Odontológica. Norma General Técnica Nº 105 In: Departamento de Salud Bucal. División de prevención y control de Enfermedades SdSP, Editor: Gobierno de Chile, Ministerio de Salud de Chile; 2008. p. 15-20.

7. Ellwood R, Ferjeskov O, Cury JA, Clarkson B. Fluorides in Caries Control. In: Kidd Fa, editor. Dental Caries, The disease and its clinical management: Oxford.; 2008. p. 288-322.

8. McDonagh MS, Whiting PF, Wilson PM, Sutton AJ, Chestnutt I, Cooper J, et al. Systematic review of water fluoridation. BMJ 2000; 321 (7265): 855-9.

9. Iheozor-Ejiofor Z, O’Malley L, Glenny AM, Macey R, Alam R, Tugwell P. Water fluoridation for the preven- 
tion of dental caries. The Cochrane Collaboration. 2015; p. 30 .

10. Dean HT. Fluorine and dental caries. Am J Orthod 1947; 33 (2): 49-67.

11. Cury J. Uso do fluoruro e controle da Cárie como Doença. En: Baratieri, editor. Odontologia restauradora-Fundamentos e Possibilidades; São Paulo, Brasil. Ed. Santos; 2001. p. 31-68.

12. Warren JJ, Levy SM, Broffitt B, Cavanaugh JE, Kanellis MJ, Weber-Gasparoni K. Considerations on optimal fluoride intake using dental fluorosis and dental caries outcomes a longitudinal study. J Public Health Dent 2009; 69: 111-5.

13. Peckham S, Awofeso N. Water fluoridation: a critical review of the physiological effects of ingested fluoride as a public health intervention. The Scientific World JOURNAL 2014; 293019.

14. Soto L, Tapia R, Jara G, Rodríguez G, Urbina T, Venegas C, et al. Diagnóstico Nacional de Salud Bucal del Adolescente de 12 años y Evaluación del Grado de Cumplimiento de los Objetivos Sanitarios de Salud Bucal 2000-2010. Chile 2007.

15. Danielson C, Lyon JL, Egger M, Goodenough GK. Hip fractures and fluoridation in Utah's elderly population. JAMA 1992; 268: 746-8.

16. Kurttio P, Gustavsson N, Vartiainen T, Pekkanen J. Exposure to natural fluoride in well water and hip fracture: a cohort analysis in Finland. Am J Epidemiol 1999; 150: 817-24.

17. Sowers MR, Clark MK, Jannausch ML, Wallace RB. A Prospective study of bone mineral content and fracture in communities with differential fluoride exposure. Amer J Epidemiol 1991; 133: 649-60.

18. Li Y, Liang C, Slemenda CW, Ji R, Sun S, Cao J, et al. Effect of long-term exposure to fluoride in drinking water on risks of bone fractures. Bone Miner Res 2001; 16: 932-9.

19. Riggs BL, Hodgson SF, O'Fallon WM, Chao EY, Wahner HW, Muhs JM, et al. Effect of fluoride treatment on the fracture rate in postmenopausal women with osteoporosis. N Engl J Med 1990; 322: 802-9.

20. Simonen O, Laitinen O. Does fluoridation of drinking-water prevent bone fragility and osteoporosis? Lancet 1985; (2): 432-4.

21. Park EY, Hwang SS, Kim JY, Cho SH. [Effects of longterm fluoride in drinking water on risks of hip fracture of the elderly: an ecologic study based on database of hospitalization episodes]. J Prev Med Public Health 2008; 41 (3): 147-52.

22. Yin XH, Huang G-L, Lin DR, Wan CC, Wang YD, Song $\mathrm{JK}$, et al. Exposure to Fluoride in Drinking Water and
Hip Fracture Risk: A Meta-Analysis of Observational Studies. PLoS ONE 2015; 10 (5).

23. Manocha SL, Warner H, Olkowski ZL. Cytochemical response of kidney, liver and nervous system of fluoride ions in drinking water. Histochemical J 1975; 7: 343-55.

24. $\mathrm{Hu} \mathrm{YH}, \mathrm{Wu}$ SS. Fluoride in cerebrospinal fluid of patients with fluorosis. J Neurol Neurosurg Psychiatry 1988; 51: 1591-3.

25. Chan AW, Minski MJ, Lai JC. An application of neutron activation analysis to small biological samples: simultaneous determination of thirty elements in rat brain regions. J Neurosci Methods 1983; 7: 317-28.

26. Zhu W, Zhang J, Zhang Z. Effects of fluoride on synaptic membrane fluidity and PSD-95 expression level in rat hippocampus. Biol Trace Elem Res 2011; 139: 197-203.

27. Reddy PY, Reddy KP, Kumar KP. Neurodegenerative changes in different regions of brain, spinal cord and sciatic nerve of rats treated with sodium fluoride. J Med Allied Sci 2011; 1 (1): 30-5.

28. Still CN, Kelley P. On the incidence of primary degenerative dementia vs. water fluoride content in South Carolina. Neurotoxicology 1980; 1: 125-31.

29. Haimanot RT. Neurological Complications of Endemic Skeletal Fluorosis, with Special Emphasis on Radiculo-Myelopathy Paraplegia 1990; 28 (4): 244-51.

30. Choi AL, Sun G, Zhang Y, Grandjean P. Developmental fluoride neurotoxicity: a systematic review and meta-analysis. Environ Health Perspect 2012; 120: 1362-8.

31. Shivaprakash PK, Ohri K, Noorani H. Relation between dental fluorosis and intelligence quotient in school children of Bagalkot district. J Indian Soc Pedod Prev Dent 2011; 29: 117-20.

32. Ding Y, Yanhui G, Sun H, Han H, Wang W, Ji X. The relationships between low levels of urine fluoride on children's intelligence, dental fluorosis in endemic fluorosis areas in Hulunbuir, Inner Mongolia, China. J Hazard Mater 2011; 186: 1942-6.

33. Broadbent JM, Thomson WM, Moffitt TE, Poulton R. Health effects of water fluoridation: a response to the letter by Menkes et al. NZ Med J 2015; 128: 73-4.

34. Broadbent JM, Thomson WM, Ramrakha S, Moffitt TE, Zeng J, Foster LA, et al. Community Water Fluoridation and Intelligence: Prospective Study in New Zealand. Am J Public Health 2015; 105: 72-6.

35. Public Health England. Water Fluoridation. Health monitoring report for England 2014. PHE publications gateway number: 2013547, 2014.

36. National Research Council (NRC). Fluoride in drinking water: a scientific review of EPA's standards. Washington DC: National Academies Press, 2006. 
37. Feltman R, Kosel G. Prenatal and postnatal ingestion of fluorides. Fourteen years of Investigation-final report. J Dent Med 1961; 16: 190-9.

38. Galletti P, Joyet G. Effect of fluorine on thyroidal iodine metabolism in hyperthyroidism. J Clin Endocrinol Metab 1958; 18: 1102-10.

39. US Public Health Services. "Review of Fluoride Benefits and Risks", Table 11, p. 17. 1991. http://health.gov/ environment/ReviewofFluoride/ (accessed 8 Jul 2014).

40. European Commission. Critical review of any new evidence on the hazard profile, health effects, and human exposure to fluoride and the fluoridating agents of drinking water. Scientific Committee on Health and Environmental Risks (SCHER), 2011.

41. Warren J, Levy S, Broffitt B, Cavanaugh JE, Kanellis MJ, Weber-Gasparoni K. Considerations on optimal fluoride intake using dental fluorosis and dental caries outcomes-a longitudinal study. J Public Health Dent 2009; 69: 111-5.

42. Day T, Powell-Jackson P. Fluoride, water hardness, and endemic goitre. Lancet 1972; 1: 1135-8.
43. Peckham S, Lowery D, Spencer S. Are fluoride levels in drinking water associated with hypothyroidism prevalence in England? A large observational study of GP practice data and fluoride levels in drinking water. J Epidemiol Community Health 2015; 69 (7): 619-24.

44. Prado E, Wurtz T, Ferbus D, Shabana el-H, Forest N, Berdal A. Sodium fluoride influences the expression of keratins in cultured keratinocytes.Cell Biology Toxicol 2011; 27 (1): 69-81.

45. Blasik LG, Spencer SK. Fluoroderma. Arch Dermatol 1979; 115 (11): 1334-5.

46. Cheng KK, Chalmers I, Sheldon T. Adding fluride to water supplies. BMJ 2007; 335 (7622): 699-702.

47. Milk fluoridation for the prevention of dental caries. World Health Organization. 2009. Pag. 41-5.

48. Zillmann Geerdts G. Prevalencia de caries en niños de 8 años que residen desde su nacimiento en zonas no fluoruradas y asisten a escuelas con y sin programa de alimentación escolar fluorurado (PAE-F.) Universidad de Chile, Facultad de Odontología, Departamento de Odontopediatría, 2010 (tesis) p 53-4. 